\title{
Possible Risks of Human Tumor Virus Infection among Overseas Travelers in Japan
}

\author{
Michiko Yamamoto ${ }^{1}$ and Naoki Yamamoto ${ }^{2 *}$ \\ ${ }^{1}$ Department of Drug Informatics, Center for Educational \& Research Center on \\ Clinical Pharmacy, Showa Pharmaceutical University, Japan \\ ${ }^{2}$ Department of Molecular Virology, Tokyo Medical and Dental University, Japan
}

\section{Mini Review \\ Volume 2 Issue 1}

Received Date: March 26, 2018

Published Date: April 05, 2018

*Corresponding author: Naoki Yamamoto, Department of Molecular Virology, Tokyo Medical and Dental University, Tokyo, Japan, Email: nyamamoto0508@gmail.com

\section{Abstract}

Under the recent trend of rapid increase of overseas travelers in Japan, the potential risks of visitors to the human tumor virus infections (HTLV-I, high -risk HPVs, HBV, HCV and EBV) were summarized based on original research/review articles and recent surveillance reports. Almost all human tumor virusesare transmitted through blood, mother to child and sexual contact. Amongst them, only infection with HBV and HPVs are vaccine-preventable. However, HPV vaccination coverage is very low in Japan when compared to western developed countries due to several complicated reasons. While HTLV-I is uniquely prevalent in Japan. Thus, most human tumor virus infections represent the most likely risks for overseas travelers in Japan. Maintaining an up-to-date routine vaccination schedule is therefore highly recommended, especially those who attend the Tokyo 2020 Olympic Games and the Paralympic Games. Also, it is very important for them to take action to prevent human tumor virus infections by using self-protective measures such as condom. Infection risk of HCV and EBV is considered low in Japan.

Keywords: Human tumor viruses; Travelers; Japan; Epidemiology; Mode of transmission; Vaccines

Abbreviations: BL: Burkitt's lymphoma; NPC: Nasopharyngeal cancer; SC: Stomach cancer; CAEBV: Chronic active EBV; ENKTL: Extranodal NK/T cell lymphoma; ANKL : Aggressive NK leukemia; IM: Infectious Mononucleosis; EBV-LPD: EBV-associated lympho-proliferative diseases; HCC: Hepato-cellular carcinoma; CLD: Chronic Hepatic Diseases; ATLL: Adult Tcell leukemia/ lymphoma; HAM/TSP; HTLV-I-associated myelopathy/ tropical spastic paraparesis; CC: Cervical Cancer; AGC: Ano-Genital Cancer; OPC: Oro-pharyngeal cancer; MTC: Mother to child transmission; Non-sexual transmission includes intravenous drug use, and behaviors associated with tattooing and piercing.

\section{Introduction}

Tumor viruses are viruses that can cause cancer, and are also called on coviruses or cancer viruses [1]. They include several types of DNA and RNA viruses, and in man, seven viruses such as Epstein-Barr virus (EBV), human Tlymphotropic virus type 1 (HTLV-I), hepatitis B virus (HBV), human papilloma viruses (HPVs), hepatitis $\mathrm{C}$ virus 
(HCV), Kaposi's sarcoma-associated herpes virus (KSHV), and Merkel cell polyomavirus (MCPV) are currently known. Tumor viruses can immortalize and transform normal cells and can also induce cancers in animals. All known human tumor viruses have the property of persistent infection, and over the years, some viral genes activate cancer-related genes involved in cell proliferation and/or inhibit tumor suppressor genes eventually leading to uncontrolled growth of the cells. About 15 percent of cancers in humans are believed to be associated with viral infections at present [2].

Travelers from foreign countries are increasing rapidly in Japan. The government has encouraged to enhance the overseas tourism and the number of foreign tourists visiting Japan has continued to grow consistently for the past 6 years except its temporary decrease due to the Great East Japan Earthquake in 2013. And indeed, 40 million travelers per year by 2020 are expected to visit Japan [3]. In this way, the Tokyo Olympic Games and the Paralympic Games (OPG) to be held in 2020 will be the symbolic event for this trend.

Needless to say, there is a close relationship between the contact frequency among humans and epidemics of infectious diseases. Contact with humans by travel, especially overseas travel, promotes opportunities for people to encounter new pathogens and infectious diseases caused by them. This includes basically all infectious diseases acquired via air-borne infection, contact infection, and sexually transmitted infections.

In Japan, some vaccines have not been used efficiently though they are available. For example, in the past, measles outbreak took place repeatedly in Japan. However, thanks to the development of effective vaccines and their high coverage, it was eliminated in 2015 once. Then, as a matter of fact, the vaccination rate has declined gradually, and several imported cases had been reported [4]. Furthermore, even exported measles cases and outbreaks initiated by Japanese travelers have been reported from the United States and other countries ironically [5]. It is unknown whether this is due to an excessive sense of security that Japanese people no longer need to worry about measles.

According to Nakamura et al. who summarized the potential health risks for visitors to Tokyo OPG very recently, communicable diseases for vaccine-preventable illnesses such as measles and rubella, as well as food and waterborne diseases represent the most likely risks [6]. While the risk of acquiring vector-borne diseases is considered low in Japan. Visitors attending the OPG might import these diseases into Japan, causing epidemics during this time. They recommended, therefore, that all visitors attending Tokyo 2020 should ensure that their routine vaccinations are up-to-date.

Possible risks of human tumor virus infections for overseas travelers in Japan Possible risks of tumor virus infection for overseas travelers and those in contact with them are summarized in Table 1.In general, there is a close connection between travel and sexually transmitted diseases, and most of these tumor viruses are transmitted by sexual activities. Actions that allow penetration of virus-infected cells into body (breast feeding and unprotected sexual contacts) are likely to enhance infection risk. HTLV-I and HPV infection may be infectious diseases of particular concern to tourists visiting Japan. These topics are discussed based on original research/review articles and recent surveillance reports, however, discussion for KSHV and MCPV is omitted here due to the lack of sufficient data available in Japan. Epidemiological features for these cancer viruses and infection risks during traveling in Japan are reviewed as follows one by one.

\section{HTLV-I}

Since many HTLV-I carriers and related diseases are present in southwest Japan including Kyushu-Okinawa region, Japan is the only country endemic for HTLV-I among developed countries. Blood and body fluids containing visible blood, semen and vaginal secretions represent a risk of transmission of HTLV-I [7]. Though most retroviruses can primarily infect in the manner of cell-free as well as cell-to-cell infection, HTLV-I can be transmitted solely by cell-cell interactions [8]. In HTLV transmission, infected lymphocytes in the breast milk, semen, and blood are considered to be crucial. In fact, it is estimated that infection between married couple takes place in about $20 \%$ of women 2 years after marriage if men are carriers and women are not. However, when infected after being adults, infected persons are believed to hardly develop ATLL fortunately. Nevertheless, infecting HTLV-I by sexual intercourse means that the victims become virus carriers themselves and next, the virus will be transmitted to his/her sexual partners.

To estimate the nationwide annual number of new HTLV-1 infections, more than 3 million HTLV-Iseronegative blood donors were investigated by Satake, et al. [9]. They found that 532 people (204 men and 328 women) had seroconvert in a median follow-up period of 
$4 \cdot 5$ years. The incidence density was significantly higher in women $(6 \cdot 88 / 100000$ person/years) than in men $(2$ - $29 / 100000$ person/years). The estimated annual number of new HTLV-I infections in Japan was 4190 with 975 infections in men and 3215 in women. Although infection to women from men is dominant it is important to note that infection from women to men also occurs with considerable frequency.

During its long journey for evolution, HTLV-I seemed to select a strategy to stay long in the living organisms instead of obtaining its weak oncogenicity. Longer survival in the body of carriers will provide the virus more chance to infect other individuals. It is important that there is the possibility of spreading infection from an asymptomatic carrier to his or her spouse during this time. Particularly important fact in mother - to - child infection is that ATLL develop only when children are infected from infected mothers vertically at early childhood. After virus infection, a long period of 30 to 70 years is necessary until the actual symptoms appear, and it is estimated that the probability of the disease onset is about $5 \%$ (one in 20 infected people) [10]. Currently, mother-to-child transmission is prevented by receiving a screening test at the time of pregnancy. Similarly, infection by blood transfusion is thought to have ceased now due to the introduction of viral screening at the time of blood donation.

\section{HPVs}

HPV viridae is a large viral family composed of many serotypes . HPV is mainly transmitted sexually. Also, infants, children and adults can acquire infections by birth or by close contact. High-risk HPVs causing cervical cancer are often eliminated naturally by the host immunity, and accordingly, HPVs can infect multiple times. Currently, two types of vaccines are available in Japan; Cervarix has effects on two types of high-risk HPV, HPV-16 and 18 while Gardasil 4 is effective against two other types of HPVs which are the cause of genital warts in addition to HPV16 and 18 [11]. Gardasil 9 covering HPV $6 、 11,16 、 18,31,33 、 45 、 52$, and 58 has not yet been approved in Japan at present.

Infection rates of corresponding HPV types in women were decreased significantly by introduction of HPV vaccines in developed countries [12]. In Australia, the infection rates of vaccine type HPVs in women aged 18-24 years decreased markedly after introduction of the HPV vaccination program. Importantly, the infection decreased also in women who were not vaccinated, suggesting that the herd immunity effect was exerted [13]. In Japan, Tanaka and colleagues found that percentage of people judged to be at least ASC-US (atypical squamous cells of undetermined significance which requires precise examination) or higher is decreased significantly by about $80 \%$ compared with the non-vaccinated women [14]. They also confirmed that these high preventive effects of vaccines lasted long (8 years or 9.4 years). Despite these evidences, the increase in mortality due to cervical cancer is rather accelerating in Japan when looking at the rate of change in cancer age-adjusted mortality rate (under 75 years old).

In Japan, the immunization law was revised in 2013, and periodic vaccination of HPV vaccines using Gardasil and Cervarix was started. Vaccine coverage rates for the HPV vaccine have reached as high as 70 percent in 2013. Then, it rapidly plummeted to less than 1 percent today due to the occurrence of adverse events such as chronic pain and other symptoms after vaccine implementation, and active promotion of periodic vaccination is now suspended [15]. In response to this attitude of the Japanese Government, the World Advisory Committee on the Safety of WHO Vaccines (GACVS) issued a statement criticizing this based on the clinical data review performed by the National Expert Committee in Japan that concluded that these symptoms were not related to the vaccines [16]. Nevertheless, we could not reach agreement on resumption of recommendation for HPV vaccination temporarily. It is remarkable that although the vaccine is available and infection prevention is possible for HPV, its vaccination coverage is very low in Japan when compared to western developed countries. Thus, though epidemiological situation is also unique even in the field of human tumor viruses, its reason seems to be different from the measles case. Among Japanese people, there seems to be a complicated psychological background including distrust of the pharmaceutical industries, a history of vaccine confidence issues regarding the safety and knowledge-gap about HPV vaccines [17].

As a result, young women are left to be exposed to the risks of HPV-related cancers that can be prevented. Due to insufficient evidence-based policy decision, failure to use a safe and effective vaccine is condemned to lead to truly harmful results. Regarding the safety of the HPV vaccine, it is different from the purpose of this paper, so we would like to refrain from further discussion. Instead, we would like to just emphasize the reason why the vaccination rate of current HPV vaccines is very low in Japan when compared with the developed countries in Europe and the
Michiko Yamamoto and Naoki Yamamoto. Possible Risks of Human Tumor Virus Infection among Overseas Travelers in Japan. J Inf Dis Trav Med 2018, 2(1): 000107. 


\section{Journal of Infectious Diseases \& Travel Medicine}

US. If HPV vaccine is indeed effective, higher vaccination coverage should result in the higher level of herd immunity and the lower viral load in the community. Thus, it is likely that the risk of infection with HPV in Japan is higher than that in developed countries with high HPV vaccination rate and that special attention is required for travelers visiting Japan. Also, it is important to note that other types of high-risk HPVs such as HPV52 and 58 are frequently detected in patients with cervical intraepithelial neoplasia and invasive cervical cancer in East Asian countries including Japan in addition to HPV 16 and 18 which are dominant in Europe and the United States [18].

\section{HBV}

Contaminated blood, semen and vaginal secretions represent risks for the vertical as well as horizontal transmission of HBV. Transfusion of blood and blood products used to be one of the most important risk factors in Japan. However, upon the HBs antigen testing started in 1972, the number of people infected with HBV by transfusion has significantly decreased, and now there is little infection risk through this route. However, the youth behaviors including repeated use of contaminated needles/syringes among drug abusers, or drilling with tattooing and piercing are still risky. Amongst all, sexual intercourse is the most important reason for HBV infection. The number of cases increased from 174 in 2006 to 236 in 2015 in Japan. Of these, 20\% of cases were reported in Tokyo and $70 \%$ of cases were acquired through sexual contact [19]. Hence, hepatitis B infection is another consideration in association with the increase of travelers. Maternal infection at birth is the most important as the reason for vertical HBV infection. Currently, Japan has 1.1 million to 1.4 million people infected with HBV [20]. In Japan, a nationwide prevention program against mother-to-infant infection by HBV started in 1985 since most of HBV infection used to be due to this infection route. In addition, it is known that the number of HBV carriers is very small in the young generation born after this HBV mother-to-child transmission prevention project was implemented. In fact, based on the data among the donor population who donated blood for the first time at the Japan Red Cross Blood Center in 2000, the HBs antigen prevalence rate was less than $1 \%$ among those who were at the age of $<40$ years, but it exceeded 1\% at 40-69 years showing that it still shows high values in elderly Japanese population [21].

\section{HCV}

Like HBV, HCV causes chronic hepatitis and HCC, and about $60 \%$ of chronic hepatitis is considered to be due to the HCV in Japan. Furthermore, about $80 \%$ of Japanese liver cancer patients arises from HCV infection. And it is estimated that about $1.5 \%$ of the population and about 2 million carriers are present in Japan [22]. Among them, about 900,000 people will develop hepatitis C, about 210,000 cirrhosis of the liver, about 15,000 cases of liver cancer, and the remaining about 700,000 people are said to be asymptomatic carriers.

Though the route of transmission of HCV is not necessarily understood well, parenteral exposure via blood or blood products is most important. There is no air-borne infection or oral infection of HCV. Many people in Japan are now believed to have been infected with this virus by blood transfusion or blood products before the discovery of HCV, or the use of contaminated needles before disposable needles had become available. The vaccine is not currently available, but risks of the new infection is to pierce or tattoo, the use of stimulant drugs, or acupuncture in unsanitary conditions. On the other hand, infection by sexual intercourse and mother-to-child infection are extremely rare, so there are few problems with travelers.

\section{EBV}

EBV is a type of herpes virus that exists very ubiquitously [23]. EBV is transmitted through body fluids such as saliva, blood and semen during child care, sexual contact, blood transfusions, and organ transplantations.EBV establishes latent infection state and is not eliminated throughout life. And the virus is activated (probably from pharyngeal epithelium) irregularly to maintain or expand.EBV causes infectious mononucleosis (IM) as a result of primary infection. Actually, when EBV was found to be a causative agent of IM for the first time, this disease was also called a kissing disease, prevalent among university students [24]. But IM is in general self-limiting terminated with the rise of antibody titer to EBV in peripheral blood. Since the virus is so common and the rate of those who actually develop IM or lympho-proliferative diseases (LPD) is not high upon EBV infection, there are aspects that are likely to be optimistic compared to other viral diseases. However, once EBV-positive cancer develops, it is often refractory and highly malignant, and thus EBV cannot be neglected. The major infected cells of EBV are B lymphocytes of the blood, but the virus can also infect other compartments of 
the blood cells such as T lymphocytes and NK cells, and epithelial cells [25]. Primary infection in infancy is asymptomatic and only a small proportion of children develop EBV-LPD. As $90 \%$ or more have already been infected in adults, the risk of EBV infection in travel may not be a big problem.

Under the recent trend of rapid increase of overseas travelers in Japan, the potential infection risks for visitors and local residents by the human tumor viruses including
EBV, HBV, HTLV-I, HPV and HCV are discussed. In consideration of the risk of virus infection during traveling, it is necessary to take epidemiological characteristics of each virus into account. No human tumor virus is transmitted through the vector, inhalation, ingestion or fomites but most of their infections are strongly associated with maternal, sexual and iatrogenic activities. Especially, sexual route (heterosexual, homosexual and oral ones) is most important for travelers as the highest risk (Table 1).

\begin{tabular}{|c|c|c|c|c|c|}
\hline Virus & Cancer (Others) & Transmission route & Prevalence & Vaccination rate & $\begin{array}{c}\text { Infection } \\
\text { risks }\end{array}$ \\
\hline EBV & $\begin{array}{c}\text { BL,NPC, SC, } \\
\text { CAEBV,ENKTL, } \\
\text { ANKL(IM, EBV-LPD) }\end{array}$ & Maternal Iatrogenic & World-wide & - & $\begin{array}{c}\text { Most adults are } \\
\text { infected. }\end{array}$ \\
\hline HBV & HCC (CLD) & $\begin{array}{c}\text { Maternal Iatrogenic } \\
\text { Sexual }\end{array}$ & High in Asia & High & Relatively high \\
\hline HTLV-I & ATLL (HAM/TSP) & Maternal Sexual & Endemic in Japan & - & Relatively high \\
\hline HPV16,18 & $\begin{array}{c}\text { CC, AGC, OPC (Genital } \\
\text { warts) }\end{array}$ & Sexual Close contact & World-wide & Low & Relatively high \\
\hline $\begin{array}{c}\text { Other high- } \\
\text { risk types }\end{array}$ & HCC (CLD) & Iatrogenic & World-wide & - & Relatively high \\
\hline HCV & Relatively low \\
\hline
\end{tabular}

Table 1: Possible tumor viral infections for overseas visitors in Japan: Viruses, cancer species, transmission route, prevalence, vaccination rate and infection risks.

Tumor virus infections are asymptomatic in general; they cause a chronic infection, and infection is basically persistent or latent, and it requires many years to decades before the disease onset or prevention effect by the vaccine has become apparent. Thus, in case of HTLV infection, it is possible that an unlucky victim infected with HTLV-I could introduce this tumor virus to his / her home land where it had not existed before without knowing the fact of infection. While in case of HPV and HBV, vaccines should be fully exploited in reducing the risk of HPV and HBV transmission to sexual partners. These vaccines can lower the infectious pool in the general population and ultimately virus-related diseases. Otherwise, once a person newly infected with HPV or HBV returns to his/her home, it may cause a change of local epidemiological situations by lowering the herd immunity level for the virus or playing a role in the epidemic locally. Especially, vaccination of youth is important in reducing the risk of HPV and HBV transmission to sexual partners.

\section{Conclusion}

Almost all human tumor viruses are transmitted through blood, mother to child infection and sexual activities. However, the most likely risk for travelers is the infection by sexual intercourse. Since only infections with HBV and HPVs are preventable by vaccines amongst human tumor viruses, maintaining an up-to-date routine vaccination schedule against them is recommended for visitors to Japan and those who attend the Tokyo 2020 OPG. While HTLV-I/ATLL is endemic in Japan, and vaccine is not available. Hence, HTLV infection represents another likely risk. Furthermore, the probability to acquire HBV is not small. Therefore, it is very important for overseas traveler to take action to prevent infection by these viruses by the use of selfprotective measures such as condom. Infection risk of $\mathrm{HCV}$ and EBV is considered low in Japan. 


\section{Journal of Infectious Diseases \& Travel Medicine}

\section{References}

1. Zur Hausen H (1999) Viruses in human cancers. Eur J Cancer 35(14): 1878-1885.

2. Zur Hausen $H$ (2009) The search for infectious causes of human cancers: where and why. Virology 392(1): 1-10.

3. (2017) Japan National Tourism Organization. Total number of visitors to Japan from foreign countries statistical data 2017.

4. National Institute of Infectious Diseases. Cumulative measles cases by week, 2010-2016 (week 1-52) (based on diagnosed week as of January 6, 2017). 2016

http://www0.nih.go.jp/niid/idsc/idwr/diseases/mea sles/measles2016/meas16-52.

5. Takahashi H, Saito H (2008) Measles exportation from Japan to the United States, 1994 to 2006. J Travel Med 15(2): 82-86.

6. Nakamura S, Wada K, Yanagisawa N, Smith DR (2018) Health risks and precautions for visitors to the Tokyo 2020 Olympic and Paralympic Games. Travel Med Infect Dis S1477-8939(18): 30005-X.

7. Yamaguchi $\mathrm{K}$, Watanabe $\mathrm{T}$ (2002) Human $\mathrm{T}$ lymphotropic virus type-I and adult T-cell leukemia in Japan. Int J Hematol 76 (2): 240-245

8. Yamamoto N, Okada M, Koyanagi $\mathrm{Y}$, Kannagi M, Hinuma Y, Et al. (1982) Transformation of human leukocytes by cocultivation with an adult $\mathrm{T}$ cell leukemia virus producer cell line. Science 217 (4561): 737-73.

9. Satake M, Iwanaga M, Sagara $Y$, Watanabe T, Okuma K, et al. (2016) Incidence of human T-lymphotropic virus 1 infection in adolescent and adult blood donors in Japan: a nationwide retrospective cohort analysis. Lancet Infect Dis 16(11): 1246-1254.

10. Ishitsuka K (2017) Progress in the management of ATL. Rinsho Ketsueki 58(6): 676-682.

11. (2008) Gardasil Shocker: Japan Withdraws Support for HPV Vaccine.

12. Lee LY, Garland SM (2017) Human papillomavirus vaccination: the population impact. Version 6: 866.

Michiko Yamamoto and Naoki Yamamoto. Possible Risks of Human Tumor Virus Infection among Overseas Travelers in Japan. J Inf Dis Trav Med 2018, 2(1): 000107.
13. Machalek DA, Chow EP, Garland SM, Wigan R, Cornall AM, et al. (2017) Human Papillomavirus Prevalence in Unvaccinated Heterosexual Men after a National Female Vaccination Program. J Infect Dis 215(2): 202208.

14. Tanaka H, Shirasawa H, Shimizu D, Sato N, Ooyama N, et al. (2017) Preventive effect of human papillomavirus vaccination on the development of uterine cervical lesions in young Japanese women. J Obstet Gynaecol Res 43(10): 1597-1601.

15. Hanley SJ, Yoshioka E, Ito Y, Kishi R (2015) HPV vaccination crisis in Japan. Lancet 385(9987): 2571.

16. (2017) Safety update of HPV vaccines. Global Vaccine Safety.

17. http://www.rho.org/files/CSIS_HPV_vac_issues_optio ns_Japan_2014.pdf

18. Tenjimbayashi Y, Onuki M, Hirose $Y$, Mori S, Ishii Y (2017) Whole-genome analysis of human papillomavirus genotypes 52 and 58 isolated from Japanese women with cervicalin traepithelial neoplasia and invasive cervical cancer. Infect Agent Cancer 12: 44.

19. (2015) Acute hepatitis B. IASR 37: 147-148.

20. Kanto T, Yoshio S (2017) Hepatitis Action Plan and Changing Trend of Liver Disease in Japan: Viral Hepatitis and Nonalcoholic Fatty Liver Disease. Euroasian J Hepatogastroenterol 7(1): 60-64.

21. https://www.niid.go.jp/niid/images/iasr/2016/08/4 38r01f01.gif

22. https://www.niid.go.jp/niid/ja/kansennohanashi/32 2-hepatitis-c-intro.html

23. Young LS, Murray PG (2003) Epstein-Barr virus and oncogenesis: from latent genes to tumours. Oncogene 22(33): 5108-5121.

24. Henle G, Henle W, Diehl V (1968) Relation of Burkitt's tumor-associated herpes-type virus to infectious mononucleosis. Proc Natl Acad Sci USA 59(1): 94-101.

25. Kasahara Y, Yachie A (2002) Cell type specific infection of Epstein-Barr virus (EBV) in EBVassociated hemophagocytic lymphohistiocytosis and chronic active EBV infection. Crit Rev Oncol Hematol 44(3): 283-294.

Copyright(C) Michiko Yamamoto and Naoki Yamamoto. 